\title{
Cardiovascular Disease in Patients with Type 2 Diabetes and in Patients Starting Empagliflozin Treatment: Nationwide Survey
}

\author{
Björn Eliasson (D) · Jan Ekelund · Rikard Amberntsson • \\ Mervete Miftaraj · Ann-Marie Svensson
}

Received: March 8, 2019 / Published online: May 14, 2019

(C) The Author(s) 2019

\begin{abstract}
Introduction: Were the participants of the EMPA-REG OUTCOME trial representative of patients receiving empagliflozin in clinical practice? The aim of the present study was to examine the prevalence of cardiovascular disease (CVD) in type 2 diabetes patients starting empagliflozin treatment in routine clinical practice in Sweden.

Methods: We used nationwide data from the Swedish National Diabetes Register (NDR), the Swedish Prescribed Drug Register, and the Swedish National Patient Register to provide clinical characteristics and ongoing treatments.
\end{abstract}

Enhanced Digital Features To view enhanced digital features for this article go to https://doi.org/10.6084/ m9.figshare. 8052392 .

Electronic supplementary material The online version of this article (https://doi.org/10.1007/s13300019-0632-4) contains supplementary material, which is available to authorized users.

B. Eliasson $(\varangle) \cdot$ A.-M. Svensson Institute of Medicine, Sahlgrenska University Hospital, University of Gothenburg, Gothenburg, Sweden

e-mail: bjorn.eliasson@gu.se

J. Ekelund · M. Miftaraj · A.-M. Svensson

National Diabetes Register, Centre of Registers, Gothenburg, Sweden

R. Amberntsson

Boehringer Ingelheim AB, Stockholm, Sweden
Results: The total study cohort included 460,558 patients, of whom 130,508 (28.3\%) had a history of CVD. The number of patients starting empagliflozin during the study period was 16,985. Among these, 1952 (11.5\%) had a history of CVD. The patients starting empagliflozin were younger than the total cohort and were more likely to have retinopathy despite having a similar duration of diabetes to the overall cohort. They also exhibited higher BMI, HbA1c, and eGFR, and were more likely to be treated with insulin and lipid-lowering and blood-pressure-lowering medications. The patients with CVD who were starting empagliflozin were slightly older and had been diabetic for slightly longer than the patients without CVD who were starting empagliflozin, but they also had lower eGFR. Among the patients with CVD who were starting empagliflozin, $87 \%$ had coronary heart disease, $8 \%$ had suffered a stroke, $13 \%$ had peripheral artery disease, 16\% had atrial fibrillation, and $20 \%$ had congestive heart failure.

Conclusion: The prevalence of CVD in patients with type 2 diabetes in clinical practice in Sweden was $28.3 \%$ during the study period, and it was $11.5 \%$ in the patients starting empagliflozin treatment. Patients of the latter cohort were, however, younger, more obese, and more likely to have unsatisfactory glycemic control, requiring additional treatment. Overall, a large proportion of type 2 diabetes patients should be considered at high cardiovascular risk. 
Funding: Boehringer Ingelheim $\mathrm{AB}$, Sweden.

Keywords: Empagliflozin; Cardiovascular disease; Prevalence; Type 2 diabetes

\section{INTRODUCTION}

Persons with type 2 diabetes mellitus (T2DM) are at elevated risk for premature death or developing cardiovascular complications. Although recent observational data show considerable improvements in cardiovascular incidence and mortality rates in persons with T2DM between 1998 and 2013, these risks are still clearly higher than in the general population [1]. Cardiovascular disease (CVD) prevention and management are thus essential parts of diabetes care [2].

The EMPA-REG OUTCOME trial examined the effects of empagliflozin, a sodium glucose cotransporter-2 (SGLT-2) inhibitor, on cardiovascular morbidity and mortality in patients with type 2 diabetes at high risk for cardiovascular events. The results showed reductions in cardiovascular death, hospitalization from heart failure, and all-cause mortality with empagliflozin treatment on top of standard care (i.e., other treatments that lower glucose, blood pressure, and blood lipids) as compared with placebo [3].

Only patients with established cardiovascular disease participated in the EMPA-REG OUTCOME trial. Are these participants representative of patients receiving empagliflozin in clinical practice, and are the results generalizable? Recently, a Swedish survey suggested that the proportion of patients with type 2 diabetes (defined as being treated with any glucose-lowering medication) in clinical practice who had cardiovascular disease (coronary heart disease, stroke, peripheral artery disease, congestive heart failure, and atrial fibrillation) was $34.2 \%$ in 2013 , implying a clearly elevated risk [4]. That study, however, lacked information on clinical characteristics and risk factors.

The aim of the present study was to examine the prevalence of CVD in patients with type 2 diabetes in routine clinical practice in Sweden- applying a similar definition to that used in the EMPA-REG OUTCOME trial-as well as the prevalence of established CVD in patients who were starting empagliflozin treatment. We used nationwide data from the Swedish National Diabetes Register (NDR) as well as the Swedish Prescribed Drug Register and the Swedish National Patient Register (National Board of Health and Welfare) to probe clinical characteristics and ongoing treatments.

\section{METHODS}

The primary objective of the study was to estimate the prevalence of a history of CVD among persons with T2DM who were alive on 31 December 2017, as well as the prevalence of a history of CVD among persons with type 2 diabetes who started empagliflozin treatment between 1 January 2015 and 31 December 2017. The secondary objective was to describe these groups of patients in terms of their clinical characteristics, including risk factors, treatments, and cardiovascular co-morbidities.

We based the study on data from the NDR, which includes approximately $90 \%$ of all patients 18 years or older who have been diagnosed with type 2 diabetes in Sweden. Health care providers report continuously directly to the NDR or via electronic patient records from routine clinical practice [5]. In the study database, the unique personal identity number assigned to each Swedish resident was used as an identifier to crosslink national healthcare registers, including the Patient Register, the Cause of Death Register, and the Prescribed Drug Register, as previously described [1]. The National Patient Register provides International Classification of Diseases (10th revision) codes associated with diagnoses made after hospitalizations and outpatient visits from 1997 on. The Prescribed Drug Register contains information on all filled prescriptions from 1 July 2005 on.

All patients with type 2 diabetes who were alive on 31 December 2017 and reported to the NDR at least once were included in the primary analysis, while all persons with type 2 diabetes who started treatment with empagliflozin between 1 January 2015 and 31 December 2017 
were included in the secondary analysis. We used data in the NDR on clinical characteristics and risk factors at baseline (including the most recent patient registration but not any patients registered more than 12 months before the index date) as well as filled prescriptions for medications lowering glucose, lipids, and blood pressure up to 12 months before the index date. All definitions of previous medical conditions before baseline are available in Table $\mathrm{S} 1$ of the Electronic supplementary material (ESM). We defined a high risk of CVD, in line with the EMPA-REG OUTCOME trial, as a history of either coronary heart disease, stroke, amputation, percutaneous coronary intervention, coronary artery bypass surgery, peripheral vascular disease, or carotid or femoral angioplasty.

HbA1c levels were reported in millimoles per mole $(\mathrm{mmol} / \mathrm{mol})$ according to the guidelines of the International Federation of Clinical Chemistry and Laboratory Medicine [9]. Microalbuminuria was defined as two positive tests from among three samples taken within 1 year, where positivity was considered to be an albu$\mathrm{min} /$ creatinine ratio of $3-30 \mathrm{mg} / \mathrm{mmol}$ ( 30-300 mg/g) or a U-albumin of $20-200 \mu \mathrm{g} /$ min (20-300 mg/L), and macroalbuminuria was considered to be an albumin/creatinine ratio of $>30 \mathrm{mg} / \mathrm{mmol}$ (>300 mg/g) or U-albumin $>200 \mu \mathrm{g} / \mathrm{min} \quad(>300 \mathrm{mg} / \mathrm{L})$ Estimated glomerular filtration rates (eGFRs) were calculated using both the Chronic Kidney Disease Epidemiology Collaboration (CKD-EPI) and Modification of Diet in Renal Disease (MDRD) equations [6, 7]. Retinal examinations were reported according to national guidelines. Physical activity (30 min walk or equivalent) was categorized into never, $<1$ time/week, 1-2 times/week, 3-5 times/week, or 5-7 times/week.

All analyses were descriptive and no testing of hypotheses was performed. For continuous variables, the distribution is described using means and standard deviations. For categorical data, frequencies and percentages are reported. We used SAS version 9.4 to analyze the data. The Regional Ethical Review Board of the University of Gothenburg approved the study, which conformed with the Declaration of Helsinki as revised in 2013, concerning human and animal rights. Springer's policy concerning informed consent has been followed. Informed consent was obtained from all individual participants included in the study.

\section{RESULTS}

The total study cohort included 460558 patients, of whom 130508 (28.3\%) had a history of CVD; see Table 1 (descriptive statistics of clinical characteristics and treatments) and Table 2 (micro- and macrovascular complications). The most marked differences between these two groups were the higher proportion of men, the higher age, the increased duration of diabetes, the higher rates of micro- and macroalbuminuria, and the lower eGFR among persons with a history of CVD. In this group, there was a history of coronary heart disease in $73 \%$, stroke in $27 \%$, peripheral artery disease in $17 \%$, atrial fibrillation in $24 \%$, and congestive heart failure in $22 \%$. Risk factor control was numerically quite similar in both groups, although the group with CVD had more treatment with insulin and with lipid- and bloodpressure-lowering medications.

The number of patients starting empagliflozin during the study period was 16,985 (Tables 1 and 2). Among these, 1952 (11.5\%) had a history of CVD, meaning that 15,033 $(88.5 \%)$ were free of CVD. The patients who were starting empagliflozin were younger than the total cohort and were more likely to have retinopathy, despite the fact that both sets of patients showed similar durations of diabetes. They also exhibited higher body mass index (BMI), glycosylated hemoglobin (HbA1c), and eGFR levels, and were more likely to be treated with insulin and lipid- and blood-pressure-lowering medications.

The (mostly male) patients with CVD who were starting empagliflozin were slightly older and had been diabetic for slightly longer than the patients without CVD who were starting empagliflozin, but they had lower eGFR levels. Among the patients with CVD who were starting empagliflozin, $87 \%$ had coronary heart disease, $8 \%$ had suffered a stroke, $13 \%$ had peripheral artery disease, $16 \%$ had atrial fibrillation, and $20 \%$ had congestive heart failure. 
Table 1 Descriptive statistics for clinical characteristics and treatments

\begin{tabular}{|c|c|c|c|c|}
\hline \multirow[t]{3}{*}{ Variable } & \multicolumn{4}{|c|}{ Mean (SD) or proportion (\%) } \\
\hline & \multicolumn{2}{|c|}{ Persons with type 2 diabetes } & \multicolumn{2}{|c|}{$\begin{array}{l}\text { Persons with type } 2 \text { diabetes starting } \\
\text { empagliflozin }\end{array}$} \\
\hline & No history of CVD & History of CVD & No history of CVD & History of CVD \\
\hline Number & 330,050 & 130,508 & 15,033 & 1952 \\
\hline Age, years & $66.2(12.6)$ & $73.7(9.9)$ & $54.1(10.5)$ & $57.4(9.5)$ \\
\hline Gender, male & $179,043(54.2 \%)$ & $84,056(64.4 \%)$ & $9585(63.8 \%)$ & $1471(75.4 \%)$ \\
\hline BMI, $\mathrm{kg} / \mathrm{m}^{2}$ & $30.1(5.6)$ & $29.5(5.2)$ & $32.1(5.7)$ & $31.6(5.3)$ \\
\hline Smoking status, yes & $29,169(13.0 \%)$ & $9993(11.8 \%)$ & $1645(14.1 \%)$ & $210(14.1 \%)$ \\
\hline $\mathrm{HbAlc}, \mathrm{mmol} / \mathrm{mol}$ & $53.3(13.3)$ & $55.1(13.6)$ & $69.0(15.5)$ & $69.5(16.0)$ \\
\hline Duration, years & $9.3(7.5)$ & $12.1(8.9)$ & $10.9(7.2)$ & $13.0(8.2)$ \\
\hline Systolic blood pressure, $\mathrm{mm} \mathrm{Hg}$ & $134.6(15.0)$ & $133.8(16.2)$ & $135.2(15.1)$ & $132.8(16.0)$ \\
\hline Diastolic blood pressure, $\mathrm{mm} \mathrm{Hg}$ & $77.6(9.6)$ & $74.2(9.9)$ & $78.9(9.7)$ & $75.6(9.8)$ \\
\hline Total cholesterol, mmol/l & $4.7(1.1)$ & $4.2(1.1)$ & $4.5(1.1)$ & $4.1(1.2)$ \\
\hline LDL cholesterol, mmol/l & $2.6(1.0)$ & $2.2(0.9)$ & $2.5(1.0)$ & $2.1(1.0)$ \\
\hline HDL cholesterol, mmol/l & $1.3(0.4)$ & $1.2(0.4)$ & $1.1(0.3)$ & $1.1(0.3)$ \\
\hline Serum triglycerides, $\mathrm{mmol} / \mathrm{l}$ & $1.9(1.3)$ & $1.9(1.2)$ & $2.3(1.9)$ & $2.4(2.2)$ \\
\hline Serum creatinine & $79.8(31.5)$ & $94.1(48.5)$ & $73.7(19.0)$ & $81.2(21.8)$ \\
\hline $\mathrm{eGFR}$ MDRD, $\mathrm{ml} / \mathrm{min} / 1.73 \mathrm{~m}^{2}$ & $81.7(24.4)$ & $71.3(24.4)$ & $90.5(24.3)$ & $83.1(23.3)$ \\
\hline $\mathrm{eGFR}$ CKD, $\mathrm{ml} / \mathrm{min} / 1.73 \mathrm{~m}^{2}$ & $81.0(20.7)$ & $69.7(21.4)$ & $88.9(17.9)$ & $81.9(18.2)$ \\
\hline Physical activity, never & $28,770(14.01 \%)$ & $16,248(21.21 \%)$ & $1979(17.96 \%)$ & $309(22.47 \%)$ \\
\hline Physical activity, $<1$ time/week & $26,394(12.85 \%)$ & $11,917(15.56 \%)$ & $1692(15.35 \%)$ & $253(18.40 \%)$ \\
\hline Physical activity, 1-2 times/week & $36,281(17.67 \%)$ & $13,243(17.29 \%)$ & $2191(19.88 \%)$ & $257(18.69 \%)$ \\
\hline Physical activity, 3-5 times/week & $47,024(22.90 \%)$ & $14,447(18.86 \%)$ & $2434(22.09 \%)$ & $232(16.87 \%)$ \\
\hline Physical activity, 5-7 times/week & $66,866(32.56 \%)$ & $20,744(27.08 \%)$ & $2725(24.73 \%)$ & $324(23.56 \%)$ \\
\hline Diabetes treatment (not insulin) & $240,491(72.87 \%)$ & $86,114(65.98 \%)$ & $15,033(100.00 \%)$ & $1952(100.00 \%)$ \\
\hline Insulin treatment & $82,250(24.92 \%)$ & $49,045(37.58 \%)$ & $6099(40.57 \%)$ & $1091(55.89 \%)$ \\
\hline Lipid-lowering treatment & $184,292(55.84 \%)$ & $105,059(80.50 \%)$ & $11,130(74.04 \%)$ & $1851(94.83 \%)$ \\
\hline Blood-pressure-lowering treatment & $243,890(73.89 \%)$ & $122,621(93.96 \%)$ & $12,037(80.07 \%)$ & $1922(98.46 \%)$ \\
\hline
\end{tabular}

$C V D$ cardiovascular disease, $S D$ standard deviation, $Q 1, Q 3$ interquartile range

Again, risk factor control was numerically quite similar for these groups of patients starting empagliflozin, although the group with CVD were more likely to be treated with insulin, and almost all received lipid-lowering and bloodpressure-lowering medications (95\% and 98\%, respectively). 
Table 2 Micro- and macrovascular complications

\begin{tabular}{|c|c|c|c|c|}
\hline \multirow[t]{3}{*}{ Variable } & \multicolumn{4}{|c|}{ Mean (SD) or proportion (\%) } \\
\hline & \multicolumn{2}{|c|}{ Persons with type 2 diabetes } & \multicolumn{2}{|c|}{$\begin{array}{l}\text { Persons with type } 2 \text { diabetes } \\
\text { starting empagliflozin }\end{array}$} \\
\hline & $\begin{array}{l}\text { No history of } \\
\text { CVD }\end{array}$ & History of CVD & $\begin{array}{l}\text { No history of } \\
\text { CVD }\end{array}$ & $\begin{array}{l}\text { History of } \\
\text { CVD }\end{array}$ \\
\hline Microalbuminuria & $40,036(19.91 \%)$ & $22,855(30.30 \%)$ & $2624(24.63 \%)$ & $430(33.26 \%)$ \\
\hline Macroalbuminuria & $7244(3.64 \%)$ & $5632(7.50 \%)$ & $522(5.10 \%)$ & $110(9.11 \%)$ \\
\hline Retinopathy & $52,714(26.02 \%)$ & $26,861(34.06 \%)$ & $3933(35.26 \%)$ & $587(43.81 \%)$ \\
\hline Amputation & - & $3308(2.53 \%)$ & & $18(0.92 \%)$ \\
\hline Coronary heart disease & - & $95,770(73.38 \%)$ & & $1694(86.78 \%)$ \\
\hline Acute myocardial infarction & - & $45,820(35.11 \%)$ & & $361(18.49 \%)$ \\
\hline Unstable angina & - & $65,459(50.16 \%)$ & & $586(30.02 \%)$ \\
\hline Percutaneous coronary intervention & - & $42,546(32.60 \%)$ & & $414(21.21 \%)$ \\
\hline Coronary artery bypass surgery & - & $18,246(13.98 \%)$ & & $77(3.94 \%)$ \\
\hline Cardiac arrest (nonfatal) & $302(0.09 \%)$ & $1133(0.87 \%)$ & $7(0.05 \%)$ & $12(0.61 \%)$ \\
\hline Stroke & - & $35,737(27.38 \%)$ & & $148(7.58 \%)$ \\
\hline Hemorrhagic stroke & $1010(0.31 \%)$ & $1209(0.93 \%)$ & $3(0.02 \%)$ & $1(0.05 \%)$ \\
\hline Transient ischemic attack & $8453(2.56 \%)$ & $11,003(8.43 \%)$ & $33(0.22 \%)$ & $31(1.59 \%)$ \\
\hline Peripheral vascular disease & $561(0.17 \%)$ & $22,114(16.94 \%)$ & $3(0.02 \%)$ & $254(13.01 \%)$ \\
\hline $\begin{array}{l}\text { Carotid or femoral angioplasty (not } \\
\text { angiogram) }\end{array}$ & - & $4736(3.63 \%)$ & & $14(0.72 \%)$ \\
\hline Atrial fibrillation & $26,748(8.10 \%)$ & $31,345(24.02 \%)$ & $455(3.03 \%)$ & $322(16.50 \%)$ \\
\hline Heart failure & $13,563(4.11 \%)$ & $29,096(22.29 \%)$ & $311(2.07 \%)$ & $390(19.98 \%)$ \\
\hline High risk of cardiovascular disease & - & $130,508(100.00 \%)$ & & $1952(100.00 \%)$ \\
\hline
\end{tabular}

$C V D$ cardiovascular disease, $S D$ standard deviation, $Q 1, Q 3$ interquartile range

\section{DISCUSSION}

This nationwide survey showed that patients starting empagliflozin treatment were relatively young compared with the overall cohort of T2DM patients, and were generally more obese and more likely to have unsatisfactory HbA1c levels. In addition, retinopathy, micro- and macroalbuminuria, and smoking were quite common in the T2DM patients starting empagliflozin treatment, in agreement with their long mean duration of diabetes ( $>10$ years). Thus, a large proportion of the patients in this nationwide cohort-even those with no history of cardiovascular disease-would qualify as high-risk individuals in recent major cardiovascular outcome trials using SGLT-2 inhibitors or GLP1-1 receptor agonists $[8,9]$.

In the multinational EMPA-REG OUTCOME trial, $71 \%$ of the participants randomized to empagliflozin treatment were male, and this cohort presented the following mean values: 
age 63.1 years, BMI $30.6 \mathrm{~kg} / \mathrm{m}^{2}$, $\mathrm{HbA} 1 \mathrm{c}$ $65 \mathrm{mmol} / \mathrm{mol}$, blood pressure 135.3/ $76.6 \mathrm{mmHg}$, LDL cholesterol $2.2 \mathrm{mmol} / \mathrm{l}$, and eGFR $74.2 \mathrm{ml} / \mathrm{min} / 1.73 \mathrm{~m}^{2}$ (MDRD) [3]. $48.0 \%$ of the patients in the pooled empagliflozin group were on insulin treatment, $94.9 \%$ were on blood pressure-lowering treatment, and $81.5 \%$ were on blood lipid-lowering therapy. Almost all of the patients in the empagliflozin group had cardiovascular disease (99.4\%), $75.6 \%$ had coronary heart disease, $23.1 \%$ had a history of stroke, $21.0 \%$ had peripheral artery disease, and 9.9\% had congestive heart failure.

The Swedish patients with CVD who were starting empagliflozin were quite similar to the participants in the EMPA-REG OUTCOME trial. The patients with no history of CVD were younger, slightly more obese, and had higher HbA1c, whereas the patients with a history of CVD showed higher levels of coronary heart disease and congestive heart failure but lower levels of stroke and peripheral artery disease than the participants in EMPA-REG OUTCOME.

A recent systematic review and meta-analysis based on the results of the EMPA-REG OUTCOME and DECLARE-TIMI 58 trials as well as the CANVAS program recently concluded that SGLT-2 inhibition should be considered in patients with type 2 diabetes regardless of the presence of atherosclerotic cardiovascular disease or a history of heart failure, due to its positive effects on glycemic control and the risks of heart failure and renal disease progression [9]. Cardiovascular benefits seem to be more pronounced in patients with established atherosclerotic cardiovascular disease, as most clearly demonstrated by the EMPA-REG OUTCOME trial [3]. The CANVAS program and the DECLARE-TIMI 58 trial, which tested the safety of canagliflozin and dapagliflozin, respectively, had larger proportions of patients at elevated cardiovascular risk, similar to the Swedish cohort, but they did not have established CVD $[10,11]$. This difference may have contributed to the outcome differences between the three trials.

The major strength of the present survey was its nationwide scope and the high validity of the data obtained, but one limitation of the study is that we did not address other comorbidities at baseline. We also used a crosssectional design and did not follow the patients longitudinally in this study. However, in a recent observational cohort study of side effects that used nationwide registers from Sweden and Denmark, we found a numerically low but increased risk of lower limb amputation and diabetic ketoacidosis in patients using SGLT-2 inhibitors compared with those on GLP1 receptor agonists, but there were no other serious adverse events of concern [12].

Other recent studies in general or high-risk populations in clinical practice have also investigated patients offered treatment with empagliflozin or other SGLT-2 inhibitors and the results of such treatment [13-16]. In essence, those studies have highlighted the positive effects as well as the safety aspects of this class of glucose-lowering medications. These effects have been attributed to several mechanisms, such as diuresis, natriuresis, and their glucose-lowering, metabolic, and renal effects [17-19].

\section{CONCLUSION}

The prevalence of CVD in patients with type 2 diabetes in clinical practice in Sweden was $28.3 \%$ during the study period, and it was $11.5 \%$ in patients starting empagliflozin treatment. Patients in the latter cohort were, however, younger and more obese and had unsatisfactory glycemic control, requiring additional treatment. Overall, a large proportion of T2DM patients should be considered at high cardiovascular risk.

\section{ACKNOWLEDGEMENTS}

Funding. The Swedish Association of Local Authorities and Regions and Region Västra Götaland fund the National Diabetes Register. Sponsorship for this study and article processing charges were funded by Boehringer Ingelheim AB, Sweden. All authors had full access to all of the data in this study and take complete 
responsibility for the integrity of the data and accuracy of the data analysis.

Authorship. All named authors meet the International Committee of Medical Journal Editors criteria for authorship for this article, take responsibility for the integrity of the work as a whole, and have given their approval for this version to be published.

Disclosures. Professor Eliasson reports personal fees (expert panels, lectures) from Amgen, AstraZeneca, Boehringer Ingelheim, Eli Lilly, Merck Sharp \& Dohme, Mundipharma, Navamedic, NovoNordisk, RLS Global, and grants and personal fees from Sanofi, all outside the submitted work. Rikard Amberntsson is employed by Boehringer Ingelheim AB, Stockholm, Sweden. Jan Ekelund, Mervete Miftaraj, and Ann-Marie Svensson have nothing to declare.

Compliance with Ethics Guidelines. The Regional Ethical Review Board of the University of Gothenburg approved the study, which conformed with the Declaration of Helsinki as revised in 2013, concerning human and animal rights. Springer's policy concerning informed consent was followed. Informed consent was obtained from all individual participants included in the study.

Data Availability. The datasets generated and/or analyzed during the current study are not publicly available due to the nature of the national quality registries, but are available from the corresponding author on reasonable request.

Open Access. This article is distributed under the terms of the Creative Commons Attribution-NonCommercial 4.0 International License (http://creativecommons.org/licenses/ by-nc/4.0/), which permits any noncommercial use, distribution, and reproduction in any medium, provided you give appropriate credit to the original author(s) and the source, provide a link to the Creative Commons license, and indicate if changes were made.

\section{REFERENCES}

1. Rawshani A, Rawshani A, Franzen S, Sattar N, Eliasson B, Svensson AM, et al. Risk factors, mortality, and cardiovascular outcomes in patients with type 2 diabetes. N Engl J Med. 2018;379(7):633-44.

2. Davies MJ, D'Alessio DA, Fradkin J, Kernan WN, Mathieu C, Mingrone $G$, et al. Management of hyperglycemia in type 2 diabetes, 2018. A consensus report by the American Diabetes Association (ADA) and the European Association for the Study of Diabetes (EASD). Diabetes Care. 2018;41(12):2669-701.

3. Zinman B, Wanner C, Lachin JM, Fitchett D, Bluhmki E, Hantel S, et al. Empagliflozin, cardiovascular outcomes, and mortality in type 2 diabetes. N Engl J Med. 2015;373(22):2117-28.

4. Norhammar A, Bodegard J, Nystrom T, Thuresson M, Eriksson JW, Nathanson D. Incidence, prevalence and mortality of type 2 diabetes requiring glucose-lowering treatment, and associated risks of cardiovascular complications: a nationwide study in Sweden, 2006-2013. Diabetologia. 2016;59(8):1692-701.

5. Eliasson B, Gudbjornsdottir S. Diabetes care-improvement through measurement. Diabetes Res Clin Pract. 2014;106(Suppl 2):S291-4.

6. Levey AS, Bosch JP, Lewis JB, Greene T, Rogers N, Roth D. A more accurate method to estimate glomerular filtration rate from serum creatinine: a new prediction equation. Modification of diet in renal disease study group. Ann Intern Med. 1999;130(6):461-70.

7. Levey AS, Stevens LA, Schmid CH, Zhang YL, Castro AF 3rd, Feldman HI, et al. A new equation to estimate glomerular filtration rate. Ann Intern Med. 2009;150(9):604-12.

8. Bethel MA, Patel RA, Merrill P, Lokhnygina Y, Buse JB, Mentz RJ, et al. Cardiovascular outcomes with glucagon-like peptide-1 receptor agonists in patients with type 2 diabetes: a meta-analysis. Lancet Diabetes Endocrinol. 2018;6(2):105-13.

9. Zelniker TA, Wiviott SD, Raz I, Im K, Goodrich EL, Bonaca MP, et al. SGLT2 inhibitors for primary and secondary prevention of cardiovascular and renal outcomes in type 2 diabetes: a systematic review and meta-analysis of cardiovascular outcome trials. Lancet. 2019;393(10166):31-9.

10. Neal B, Perkovic V, Mahaffey KW, de Zeeuw D, Fulcher G, Erondu N, et al. Canagliflozin and cardiovascular and renal events in type 2 diabetes. N Engl J Med. 2017;377(7):644-57. 
11. Wiviott SD, Raz I, Bonaca MP, Mosenzon O, Kato ET, Cahn A, et al. Dapagliflozin and cardiovascular outcomes in type 2 diabetes. $\mathrm{N}$ Engl J Med. 2019;380(4):347-57.

12. Ueda $P$, Svanstrom $H$, Melbye $M$, Eliasson B, Svensson AM, Franzen $\mathrm{S}$, et al. Sodium glucose cotransporter 2 inhibitors and risk of serious adverse events: nationwide register based cohort study. BMJ. 2018;363:k4365.

13. Cavender MA, Norhammar A, Birkeland KI, Jorgensen ME, Wilding JP, Khunti $\mathrm{K}$, et al. SGLT-2 inhibitors and cardiovascular risk: an analysis of CVD-REAL. J Am Coll Cardiol. 2018;71(22):2497-506.

14. Monami M, Dicembrini I, Mannucci E. Effects of SGLT-2 inhibitors on mortality and cardiovascular events: a comprehensive meta-analysis of randomized controlled trials. Acta Diabetol. 2017;54(1):19-36.

15. Patorno E, Pawar A, Franklin JM, Najafzadeh M, Deruaz-Luyet A, Brodovicz KG, et al. Empagliflozin and the risk of heart failure hospitalization in routine clinical care: a first analysis from the
Empagliflozin Comparative Effectiveness and Safety (EMPRISE) study. Circulation. 2019. https://doi. org/10.1161/CIRCULATIONAHA.118.039177.

16. Udell JA, Yuan Z, Rush T, Sicignano NM, Galitz M, Rosenthal N. Cardiovascular outcomes and risks after initiation of a sodium glucose cotransporter 2 inhibitor: results from the EASEL population-based cohort study (evidence for cardiovascular outcomes with sodium glucose cotransporter 2 inhibitors in the real world). Circulation. 2018;137(14):1450-9.

17. Bloomgarden $Z$. The kidney and cardiovascular outcome trials. J Diabetes. 2018;10(2):88-9.

18. Scholtes RA, van Baar MJB, Lytvyn Y, Bjornstad P, Nieuwdorp M, Cherney DZI, et al. Sodium glucose cotransporter (SGLT)-2 inhibitors: do we need them for glucose-lowering, for cardiorenal protection or both? Diabetes Obes Metab. 2019. https://doi.org/ 10.1111/dom.13692.

19. van Bommel EJ, Muskiet MH, Tonneijck L, Kramer MH, Nieuwdorp M, van Raalte DH. SGLT2 inhibition in the diabetic kidney-from mechanisms to clinical outcome. Clin J Am Soc Nephrol. 2017;12(4):700-10. 